\title{
Variations of Color with Alloying Elements in Pd-free Au-Pt-based High Noble Dental Alloys
}

Takanobu Shiraishi*, Yasuko Takuma, Eri Miura, Takeshi Fujita, Kunihiro Hisatsune

Department of Dental and Biomedical Materials Science

Unit of Basic Medical Sciences

Graduate School of Biomedical Sciences

Nagasaki University

1-7-1 Sakamoto, Nagasaki 852-8588, Japan

*Corresponding author:

Dr. Takanobu Shiraishi

Department of Dental and Biomedical Materials Science

Unit of Basic Medical Sciences

Graduate School of Biomedical Sciences

Nagasaki University

1-7-1 Sakamoto, Nagasaki 852-8588, Japan

Tel : +81-95-849-7659

Fax : +81-95-849-7658

E-mail : siraisi@nagasaki-u.ac.jp 


\begin{abstract}
The effects of alloying addition of a small amount of base metals (In, Sn, Fe, $\mathrm{Zn}$ ) on color variations in Pd-free Au-Pt-based high noble dental alloys were investigated in terms of rectilinear and polar color coordinates. The ternary Au-Pt-X $(\mathrm{X}=\mathrm{In}, \mathrm{Sn}, \mathrm{Fe}, \mathrm{Zn})$ and quaternary Au-Pt-In-Y (Y = Sn, Fe, Zn) alloys were prepared from high purity component metals. The amount of alloying base metals, $\mathrm{X}$ and $\mathrm{Y}$, were restricted up to 2 at.\%. The alloying addition of a small amount of $\mathrm{Fe}, \mathrm{In}, \mathrm{Sn}$, to a binary $\mathrm{Au}-10$ at.\% Pt alloy (referred to as AP10) effectively increased chroma, $C^{*}$. On the other hand, the addition of $\mathrm{Zn}$ to the parent alloy AP10 did not change color coordinates greatly. The increase in chroma in the present Au-Pt-based high noble alloys was attributed to the increase in the slope of spectral reflectance curve at its absorption edge near $515 \mathrm{~nm}$. It was found that the addition of a small amount of Fe to the parent alloy AP10 markedly increased lightness, $L^{*}$, and the addition of Sn gave a very light tint of red to the parent alloy. Although red-green chromaticity index $a^{*}$ contributed to chroma to some extent, contribution of yellow-blue chromaticity index $b^{*}$ was much greater in determining chroma in this Pd-free Au-Pt-based multi-component alloys. The present results are expected to be valuable in case color is to be taken into account in designing Pd-free Au-Pt-based high noble dental alloys.
\end{abstract}

\title{
Keywords
}

Gold alloy, dental alloy, optical properties, color, chroma, hue, spectral reflectance 


\section{Introduction}

Precious-metal alloys are extensively used in dentistry as restorative materials due to their good castability, appropriate mechanical properties, age-hardenability, chemical stability, and durability in the oral environment. There are several compositional types in dental precious-metal alloys, ranging from Ag-Pd-based low noble alloys to Au-Pt-based high noble alloys. Among them Au-Pt-based alloys possess the highest nobility (total amount of Au and Pt-group metals) of about 97 to 98 mass \% and are primarily used as materials for metallic core of dental porcelain-fused-to-metal restorations. In the Au-Pt-based high noble dental alloys, a small amount of base metals, such as In, Sn, Fe, Zn, are involved as alloying elements. The reason for the presence of these base metals in small quantities is the necessary precipitation hardening, as well as the ability to bond the porcelain to the metal surface [1]. The oxides of the base metals are formed at the surface of the alloy during the firing cycle and the required bond strength can be obtained by means of a very thin oxide layer of less than $1 \mu \mathrm{m}$ on the surface of the alloy [1].

Recently developed Pd-free Au-Pt-based high noble alloys are a pale yellow color. However, color of these commercial alloys varies from product to product. Considering the fact that $\mathrm{Au}$ and Pt concentrations are similar in most of the commercial Au-Pt-based high noble alloys, color variations are attributed to the elements and contents of the above-mentioned base metals. We think that color is one of the important criteria in designing gold-based dental alloys along with chemical stability, mechanical properties, and biocompatibility.

We previously prepared experimental Pd-free Au-Pt-based high noble dental alloys containing a small amount of $\mathrm{In}, \mathrm{Sn}, \mathrm{Zn}$, and measured their color coordinates, $a^{*}$ and $b^{*}$, in the three-dimensional CIE (Commission Internationale de l'Eclairage) $1976 L^{*} a^{*} b^{*}$ (CIELAB) uniform color space shown in Fig. 1 [2]. However, relationship between 
variations of color coordinates $\left(L^{*}, a^{*}, b^{*}\right)$ and concentration of the base metals added was not investigated. Further, chroma $\left(C^{*}\right)$ and hue angle $(h)$ in the polar color coordinates have not been evaluated in terms of the elements and concentrations of the base metals. Since both the $C^{*}$ - and $h$-values are important parameters in characterizing saturation of a color and hue of the material, in the present study we evaluated these color coordinates of the experimental Pd-free Au-Pt-based high noble dental alloys containing a small amount of In, $\mathrm{Sn}, \mathrm{Zn}$, and Fe. To investigate the role of chemical composition in the above-mentioned color variations, spectrophotometric colorimetry was conducted using a computer-controlled spectrophotometer.

\section{Materials and methods}

Table 1 gives chemical compositions (analyzed values in atomic percentage) of the alloys examined. A binary Au-Pt alloy (AP10) containing 10 at.\% Pt was designed as a parent alloy to evaluate the effects of various alloying base metals on the optical properties of experimental multi-component alloys. The Au-Pt-based ternary alloys containing a small amount of $\mathrm{In} / \mathrm{Sn} / \mathrm{Fe} / \mathrm{Zn}$, and quaternary alloys containing 2 at.\% In and a small amount of $\mathrm{Sn} / \mathrm{Fe} / \mathrm{Zn}$ were prepared. The amount of base metals added was restricted up to 2 at. $\%$ for the ternary alloys and 4 at.\% for the quaternary alloys. Since Pt has a strong decolorizing effect [3], a ratio of $\mathrm{Pt}$ to $\mathrm{Au}$ concentrations in atomic percentage was controlled to be constant at $1: 9$ in all the experimental alloys to highlight the effects of alloying base metals, as indicated in the right column in Table 1.

All the experimental alloys were prepared from high-purity pure metals. Appropriate amounts of constituent metals for each experimental alloy were melted in a high-frequency induction furnace. The ingots obtained were then subjected to alternate cold rolling and 
homogenizing heat-treatment at high temperatures. A number of homogenized plate samples with size of $10 \times 10 \times 0.5 \mathrm{~mm}^{3}$ were obtained.

Three pieces of the homogenized plate samples from each alloy were individually embedded in epoxy resin and subjected to mechanical grinding and polishing phases. The mechanical grinding of the samples was conducted with an automatic polishing apparatus (MA-150, Musashino Denshi Co., Ltd., Tokyo, Japan) using waterproof abrasive papers. After grinding the samples down to 2000-grit finish, they were polished with another automatic polishing apparatus (Doctor-Lap ML-180, Maruto Instrument Co., Ltd., Tokyo, Japan) using polishing cloth and alumina suspension with a grain diameter of $0.06 \mu \mathrm{m}$ finish. The mirror-polished samples were rinsed with pure water, dried, and then subjected to optical property measurements.

Optical property evaluation of the mirror-polished samples was carried out using a computer-controlled spectrophotometer (CM-3600d, Konica Minolta Sensing, Inc., Osaka, Japan). Spectral reflectance data from the mirror-polished surfaces under the illumination of the CIE standard illuminant D65 were collected with $10 \mathrm{~nm}$ intervals in the wavelengths ranging from $360 \mathrm{~nm}$ to $740 \mathrm{~nm}$. Because of the highly reflective nature of the samples, the SCI (specular component included) configuration was employed. The observer of the spectrophotometer was set to 10 -degrees. The chromaticity indices, $a^{*}$ (red-green direction), $b^{*}$ (yellow-blue direction), and lightness, $L^{*}$, in the previously described CIELAB color space in Fig. 1 were measured. Based on these coordinates, chroma, $C^{*}$, and hue angle, $h$, of the samples were also evaluated according to the following equations.

$$
\begin{gathered}
C^{*}=\left(a^{*^{2}}+b^{*^{2}}\right)^{1 / 2} \\
\tan h=b^{*} / a^{*}
\end{gathered}
$$

Figure 2 shows relationship between rectilinear $\left(a^{*}, b^{*}\right)$ and polar $\left(C^{*}, h\right)$ color coordinates. Chroma, $C^{*}$, is expressed as the distance from the origin $(\mathrm{O})$ to a point for the sample. With increasing chroma, color of the sample saturates. Hue, $h$, is the angular distance on the color 
wheel where pure red is defined as 0-degrees.

Color difference between two samples is defined as a distance between two points in the three-dimensional CIELAB color space according to the following formula:

$$
\Delta E^{*}=\left[\left(\Delta L^{*}\right)^{2}+\left(\Delta a^{*}\right)^{2}+\left(\Delta b^{*}\right)^{2}\right]^{1 / 2}
$$

where, $\Delta L^{*}, \Delta a^{*}$ and $\Delta b^{*}$ are differences in $L^{*}, a^{*}$ and $b^{*}$ coordinates, respectively, for the two samples.

\section{Results and discussion}

\subsection{Spectral reflectance curves}

Figure 3 shows typical spectral reflectance curves for the Au-Pt binary alloy AP10 and the ternary alloys AP10-X containing base metals $\mathrm{X}(\mathrm{X}=\mathrm{In}, \mathrm{Sn}, \mathrm{Fe})$ of approximately 1 at.\%. A spectral reflectance curve for pure Au was also presented for comparison. Standard deviation for three measurements at each wavelength was also shown as error bars in this figure and in the following similar figures. However, such standard deviations were so small that error bars are almost embedded in data points. For pure Au, reflectance in the long-wavelength range was very high and reflectance in the short-wavelength range was very low. This pronounced step in the spectral reflectance curve is known to cause the rich yellow color of gold. By alloying Au with 10 at.\% Pt, reflectance in the long-wavelength range markedly decreased and reflectance in the short-wavelength range considerably increased, as denoted by AP10 in Fig. 3. This marked decrease in reflectance in the long-wavelength range in the Au-Pt binary alloy was considered to be due to the formation of "virtual bound state" of electrons [3], as first suggested by Friedel [4]. By adding a small amount of base metals (In, Sn, Fe) to the parent alloy AP10, reflectance in the long-wavelength range slightly increased. Among the three base metals examined, Fe showed the greatest effects in increasing reflectance in the long-wavelength range. 
To find a wavelength at which slope of a spectral reflectance curve becomes the steepest, we investigated wavelength distribution of differential coefficient, $\mathrm{d} R / \mathrm{d} \lambda$, where $\lambda$ is wavelength and $R$ is reflectance at a given wavelength. Figure 4 shows wavelength distributions of the $\mathrm{d} R / \mathrm{d} \lambda$-values for pure $\mathrm{Au}$ and the experimental binary and ternary alloys presented in Fig. 3. It is clear that peak position of each $\mathrm{d} R / \mathrm{d} \lambda$-curve, corresponding to the absorption edge of the spectral reflectance curve shown in Fig. 3, was located near $515 \mathrm{~nm}$ regardless of alloying elements, as indicated by a double arrow. The position of the absorption edge for the experimental alloys was the same as for pure Au as seen in Fig. 4. On the other hand, peak height, corresponding to the steepness of the spectral reflectance curve, substantially varied depending on the base metals added. This effect on change in slope of the spectral reflectance curve was the strongest in Fe followed by Sn and In.

\subsection{Comparison of the effects of base metals on color variations}

Figure 5 shows distribution of chromaticity indices, $a^{*}$ and $b^{*}$, for the binary alloy AP10 and the ternary alloys AP10-X $(\mathrm{X}=\mathrm{In}, \mathrm{Sn}, \mathrm{Fe})$ described in the preceding subsection. The observed indices for pure Au were also presented for comparison. Both $a^{*}$ - and $b^{*}$-indices for the ternary alloys were noticeably larger than those for the reference alloy AP10. Hence, their data points moved slightly towards the point for pure Au. Since atomic percentage ratio of Pt to Au was controlled to be constant as presented in Table 1, the observed variations of color of the ternary alloys are clearly attributed to the base metals (In, Sn, Fe) added. By comparing Figs. 4 and 5, it is noted that with increasing steepness of the spectral reflectance curve at its absorption edge near $515 \mathrm{~nm}$, the degree of gold tinge given to a sample systematically increased.

Figures 6,7 , and 8 respectively demonstrate the effects of alloying addition of small amounts of base metals to the parent alloy AP10 on its chromaticity indices, $a^{*}$ and $b^{*}$, and lightness, $L^{*}$, in the CIELAB color space. The addition of Sn, Fe, In slightly increased the 
red-green chromaticity index $a^{*}$, demonstrating that a very light tint of red was given to the sample with the inclusion of these elements, as shown in Fig. 6. On the other hand, the addition of $\mathrm{Zn}$ did not change the $a^{*}$-value substantially. Concerning the yellow-blue chromaticity index $b^{*}$, the addition of $\mathrm{Fe}, \mathrm{Sn}$, In significantly increased the $b^{*}$-value, indicating increased yellow with the inclusion of these elements, as shown in Fig. 7. This effect was the strongest in Fe, followed by Sn and In. The effects of $\mathrm{Zn}$ addition were very weak. As can be seen in Fig. 8, the addition of Fe markedly increased lightness $L^{*}$, whereas the addition of $\mathrm{Sn}, \mathrm{In}, \mathrm{Zn}$ did not change $L^{*}$-value greatly. Summarizing these three figures, it was found that the inclusion of Fe within 2 at.\% to the Au-Pt binary alloy AP10 gave a bright yellowish gold tinge to the parent alloy. The addition of Sn or In to the alloy AP10 gave a slight gold tinge, whereas lightness of a sample was not affected greatly by these elements.

\subsection{Chroma and hue angle}

Figure 9 shows the effects of alloying addition of base metals to the parent alloy AP10 on chroma, $C^{*}$. It is clear that the effects on the increased chroma were the greatest in Fe addition followed by $\mathrm{Sn}$ and In additions. On the other hand, the addition of $\mathrm{Zn}$ did not change chroma of the parent alloy AP10 substantially. Since chroma, $C^{*}$, is expressed in the equation (1), these results are well explained by referring to the previously presented effects of the additions of these base metals on the chromaticity indices $a^{*}$ in Fig. 6 and $b^{*}$ in Fig. 7.

Figure 10 summarizes relationship between chroma, $C^{*}$, and hue angle, $h$, in the polar coordinates for all the alloys examined. It is clearly shown that chroma significantly varied with the addition of base metals. Further, chromas for the quaternary alloys were substantially larger than those for the ternary alloys. This demonstrates that dual addition of In and Fe or In and $\mathrm{Sn}$ is effective in increasing chroma of the Au-Pt-based high noble alloys. On the other hand, hue angle was not affected greatly, varying from 73.3-degrees for the alloy 
Sn2.0 to 75.8-degrees for the alloy (In2+Fe1.7). These hue angles are very close to 76.1-degrees for pure $\mathrm{Au}$, although experimental alloys contain about 10 at.\% Pt and a small amount of base metals.

Next, we investigated causes for the variation of chroma with chemical composition. In general, the perceived color of a metal is determined by the wavelength distribution of the radiation that is reflected and not absorbed [5]. Copper and gold appear red-orange and yellow, respectively, because some light photons with short wavelengths are not reflected [5]. Considering the fact that the rich yellow color of gold is caused by the pronounced step in the spectral reflectance curve, as shown in Fig. 3 and described previously, slope of a spectral reflectance curve at its absorption edge near $515 \mathrm{~nm}$ is suggested to be a reasonable indication for predicting chroma of the present Au-Pt-based high noble alloys. To examine this hypothesis, we analyzed the observed spectral reflectance curves in terms of the maximum slope at the absorption edge. Figure 11 summarizes the relationship between chroma, $C^{*}$, and maximum slope of spectral reflectance curve, $\mathrm{d} R / \mathrm{d} \lambda$, at its absorption edge for all the experimental alloys. It is clearly shown that chroma is closely related to $\mathrm{d} R / \mathrm{d} \lambda$-value and that with increasing $\mathrm{d} R / \mathrm{d} \lambda$-value $C^{*}$-value systematically increased.

Figures $12 \mathrm{a}$ and $12 \mathrm{~b}$ show relationships between chroma and red-green chromaticity index $a^{*}$ and yellow-blue chromaticity index $b^{*}$, respectively, for all the alloys examined. As $a^{*}$-value or $b^{*}$-value increased, $C^{*}$-value systematically increased. Correlation coefficients for both relations, shown in Figs. 12a and 12b, were estimated to compare strength of correlations between each chromaticity index, $a^{*}$ and $b^{*}$, and chroma, $C^{*}$. This estimation showed that a correlation coefficient between $C^{*}$ and $a^{*}$ was 0.9290 , whereas a correlation coefficient between $C^{*}$ and $b^{*}$ was 0.9997 . Therefore, it can be concluded that although a positive relationship between the chroma and the red-green chromaticity index $a^{*}$ is present, the effects of the yellow-blue chromaticity index $b^{*}$ were much greater compared to those of the $a^{*}$-value. 


\subsection{Color difference}

We evaluated color difference, $\Delta E^{*}$, between the reference alloy AP10 and the other experimental alloys according to the equation (3). The results of the evaluation are presented in Fig. 13. By comparing the $\Delta E^{*}$-values for the ternary alloys containing similar concentration (approximately 1 at.\% or 2 at.\%) of base metals, it was found that Fe has the greatest effect on color change followed by $\mathrm{Sn}, \mathrm{In}$ and $\mathrm{Zn}$. Since the $\Delta E^{*}$-value of 1.0 is said to be just discernible by the average human eye $[6,7]$, color of the alloys $\mathrm{Zn} 1.7$ and In1.0 may be scarcely distinguished from that of the reference alloy AP10. On the other hand, other ternary and quaternary alloys are considered to be distinguished from the reference alloy without difficulty.

\section{Conclusions}

The following results were obtained in the present study. This information is expected to be useful in case color is to be taken into account in designing Pd-free Au-Pt-based high noble dental alloys.

1. The addition of a small amount of Fe to a Au-Pt binary alloy was found to be most effective in giving a bright yellow color to the parent alloy.

2. The addition of a small amount of base metals with higher number of valence electrons was effective in increasing chroma of the parent Au-Pt alloy. This effect was caused by the increased slope of spectral reflectance curve at its absorption edge near $515 \mathrm{~nm}$ due to the increased reflectance in the long-wavelength range.

3. In the present Pd-free Au-Pt-based high noble alloys, chroma, $C^{*}$, was strongly dependent on yellow-blue chromaticity index $b^{*}$, and accordingly, $C^{*}$-value linearly increased with increasing $b^{*}$-value.

4. The hue angle, $h$, was not affected greatly by the alloying addition of base metals. 


\section{References}

[1] H. Knosp, R. J. Holliday and C. W. Corti: Gold Bulletin, 2003, 36(3), 93-102.

[2] T. Shiraishi, Y. Takuma, E. Miura, Y. Tanaka and K. Hisatsune: Journal of Materials Science: Materials in Medicine, 2003, 14, 1021-1026.

[3] T. Shiraishi, K. Hisatsune, Y. Tanaka, E. Miura and Y. Takuma: Gold Bulletin, 2001, 34(4), 129-133.

[4] J. Friedel: Canadian Journal of Physics, 1956, 34, 1190-1211.

[5] W. D. Callister Jr.: 'Materials Science and Engineering: An Introduction', pp. 535-536; 1985, New York, John Wiley \& Sons, Inc.

[6] R. M. German, M. M. Guzowski and D. C. Wright: Journal of Metals, 1980, 32(3), 20-27.

[7] 'Craig's Restorative Dental Materials', 12th edn, (ed. J. M. Powers and R. L. Sakaguchi), pp. 28-35; 2006, St. Louis, Mosby Elsevier. 
Figure legends and Table captions

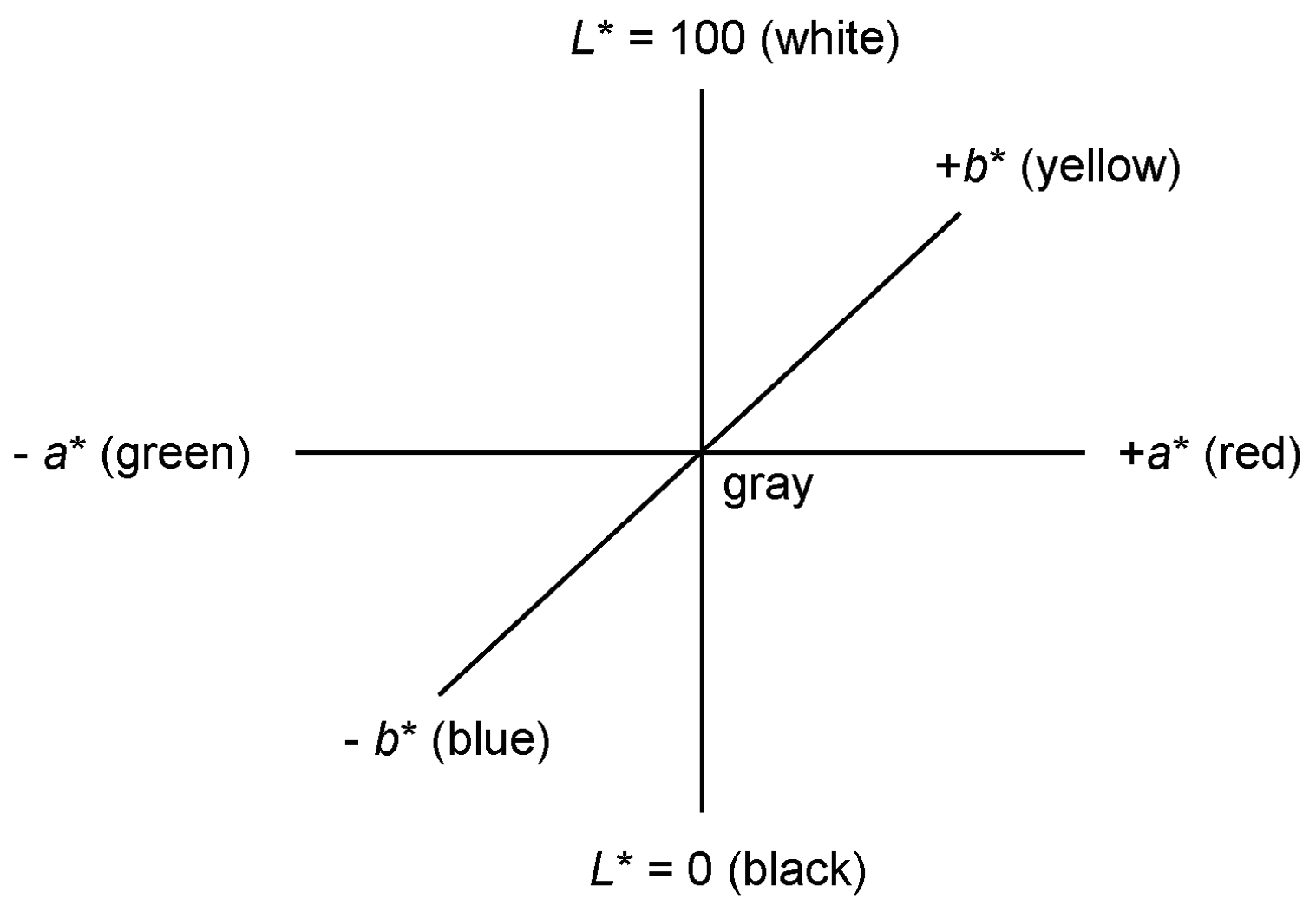

Fig. 1 Three-dimensional color coordinates in the CIE $1976 L^{*} a^{*} b^{*}$ (CIELAB) uniform color space. 


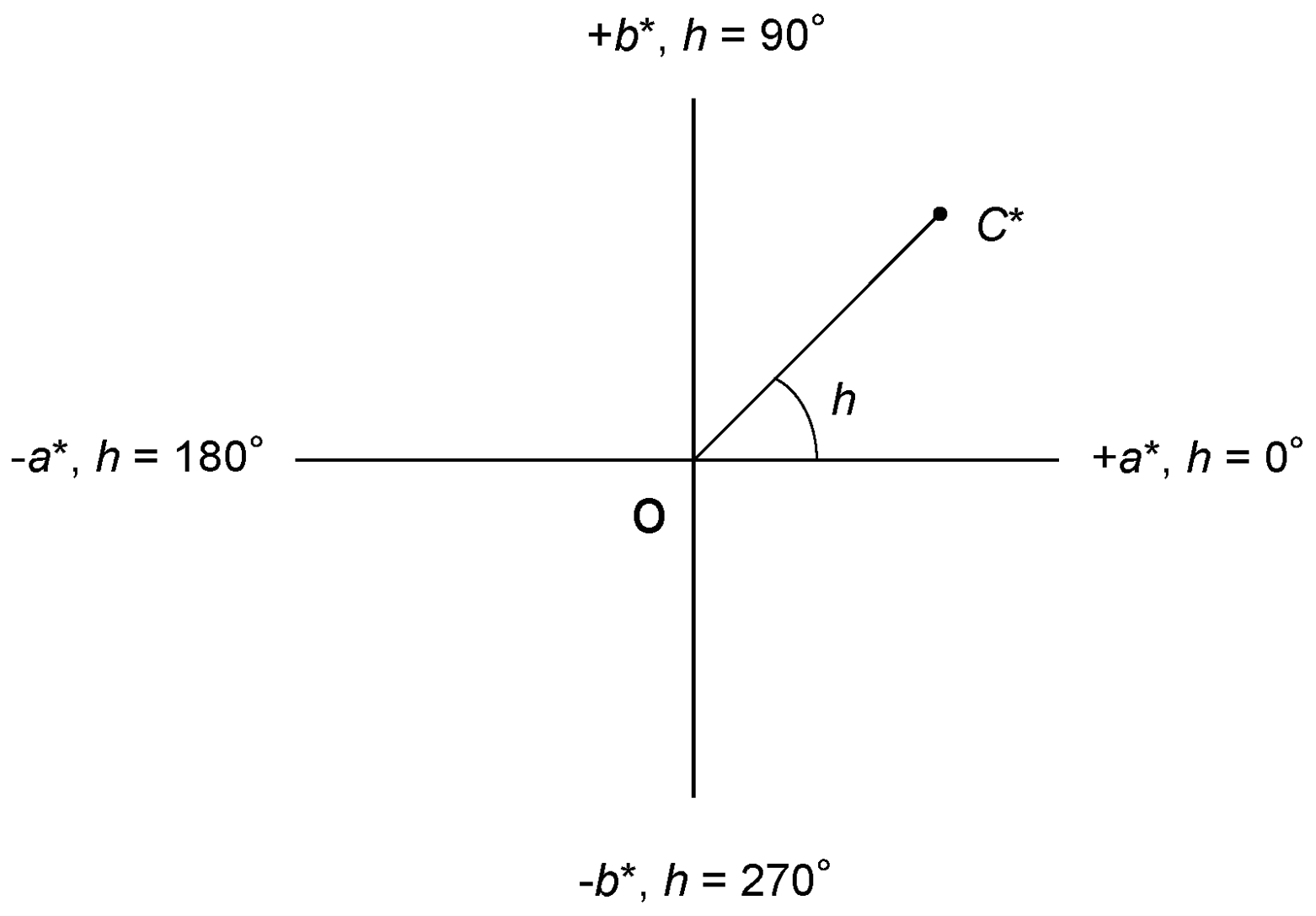

Fig. 2 Relationship between rectilinear $\left(a^{*}, b^{*}\right)$ and polar $\left(C^{*}, h\right)$ color coordinates. 


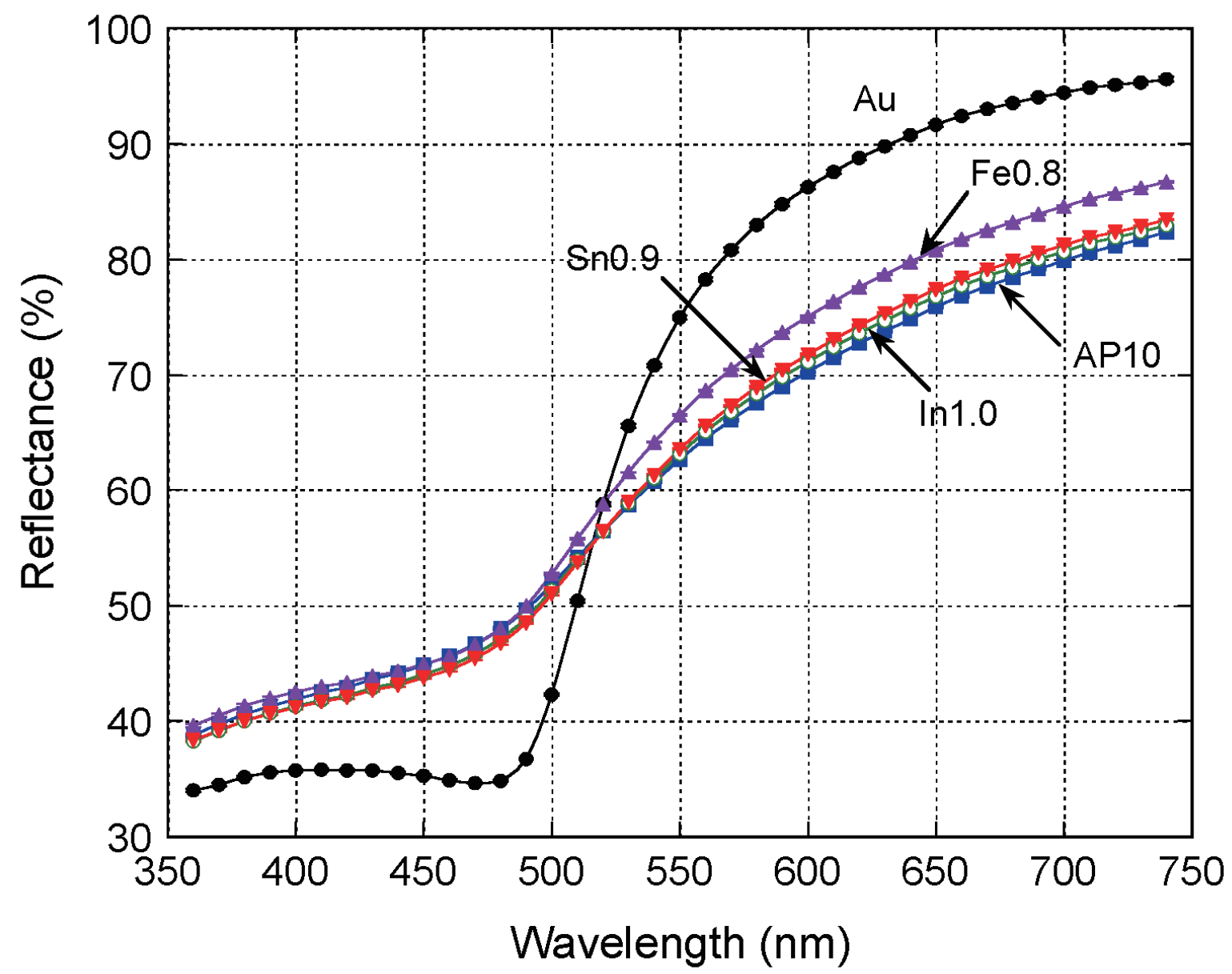

Fig. 3 Spectral reflectance curves for pure Au, the binary alloy AP10, and the ternary alloys AP10-X containing base metals $X(X=I n, S n, F e)$ of approximately 1 at. \%. 


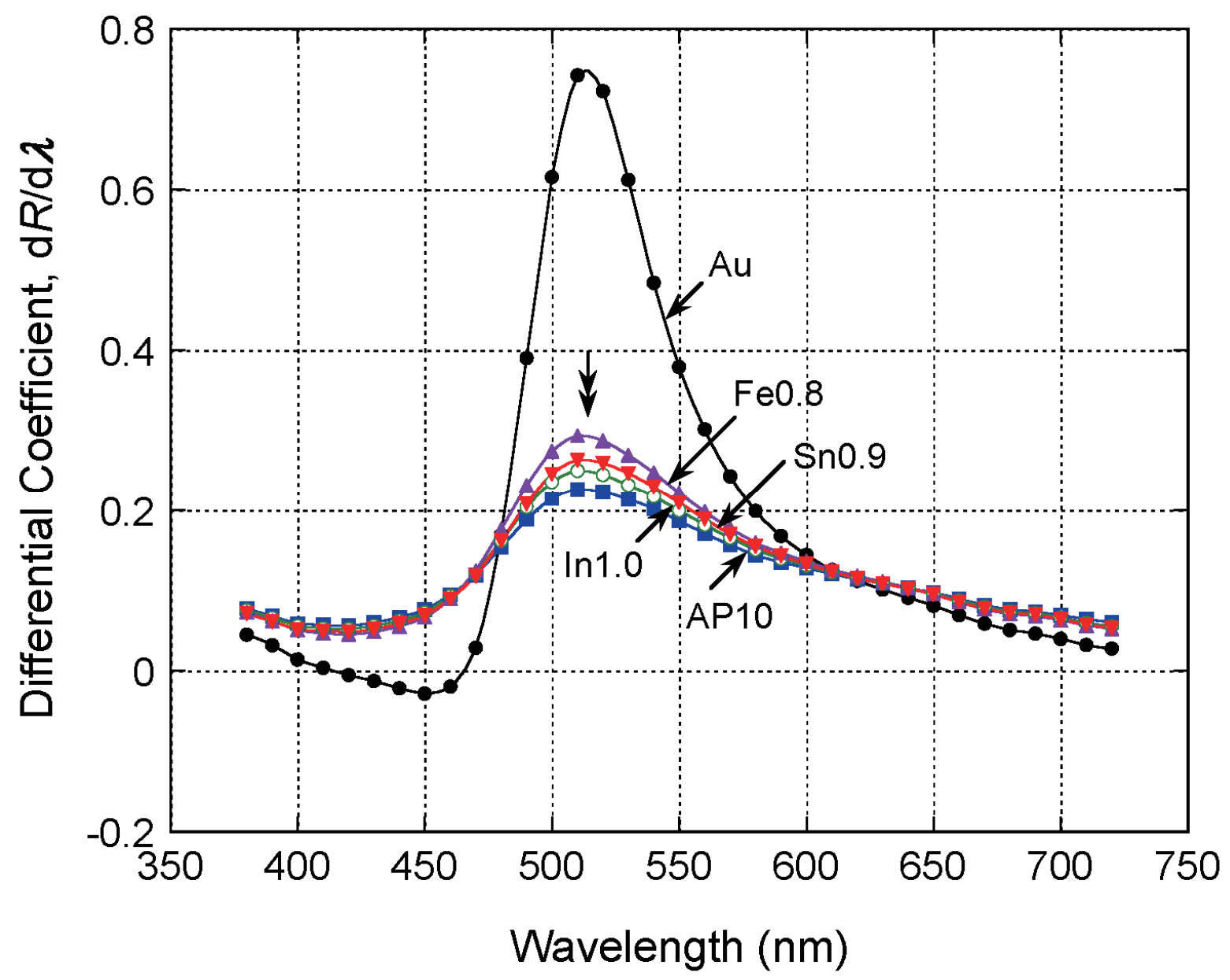

Fig. 4 Wavelength distributions of the differential coefficient, $\mathrm{d} R / \mathrm{d} \lambda$, for pure $A u$, the binary alloy AP10, and the ternary AP10-X alloys presented in Fig. 3. 


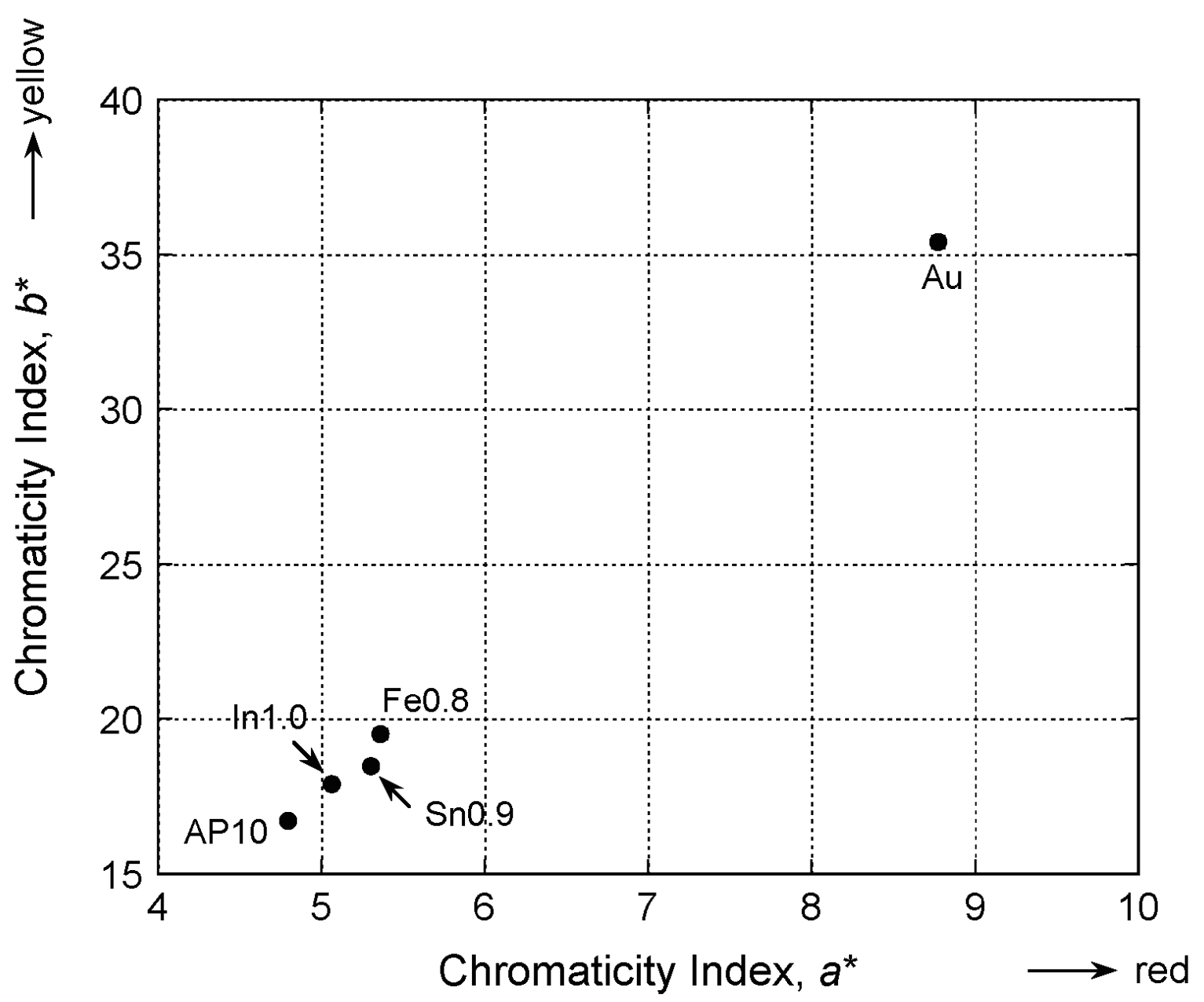

Fig. 5 Distribution of chromaticity indices, $a^{*}$ and $b^{*}$, for the binary alloy AP10 and the ternary alloys AP10-X containing base metals $X(X=I n, S n, F)$ of approximately 1 at. \%. 


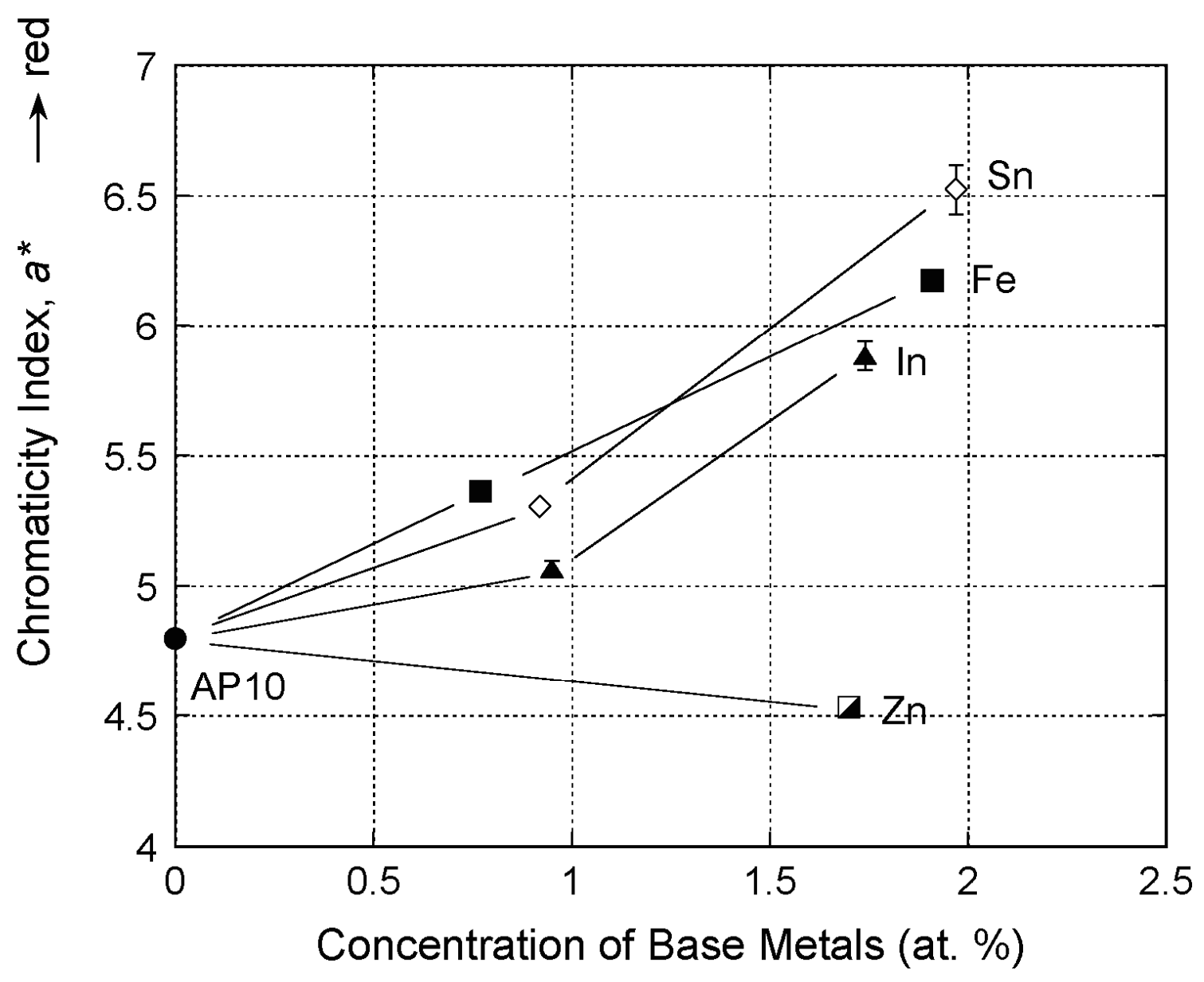

Fig. 6 The effects of alloying addition of the base metals on chromaticity index $a^{*}$ of the binary alloy AP10. 


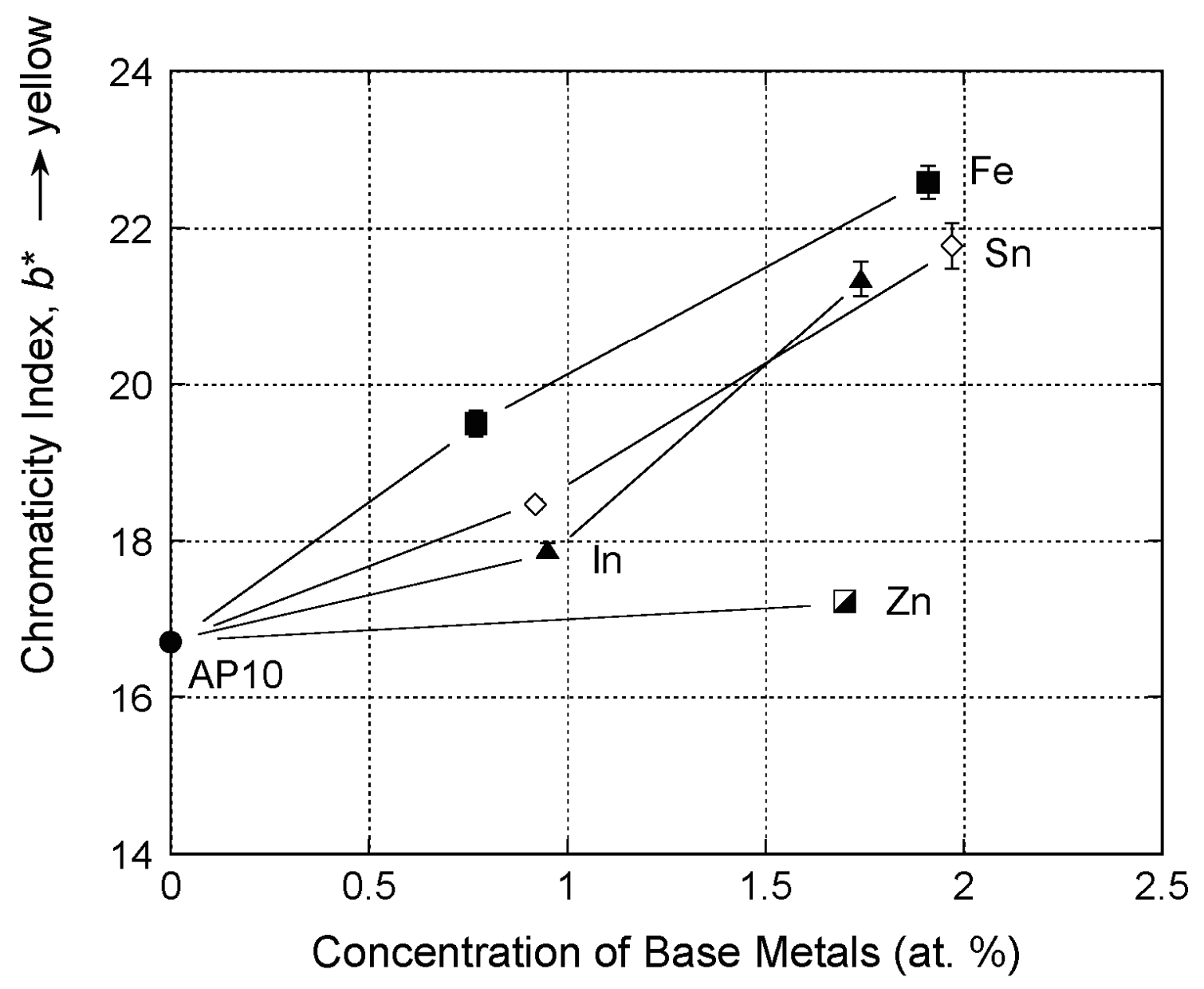

Fig. 7 The effects of alloying addition of the base metals on chromaticity index $b^{*}$ of the binary alloy AP10. 


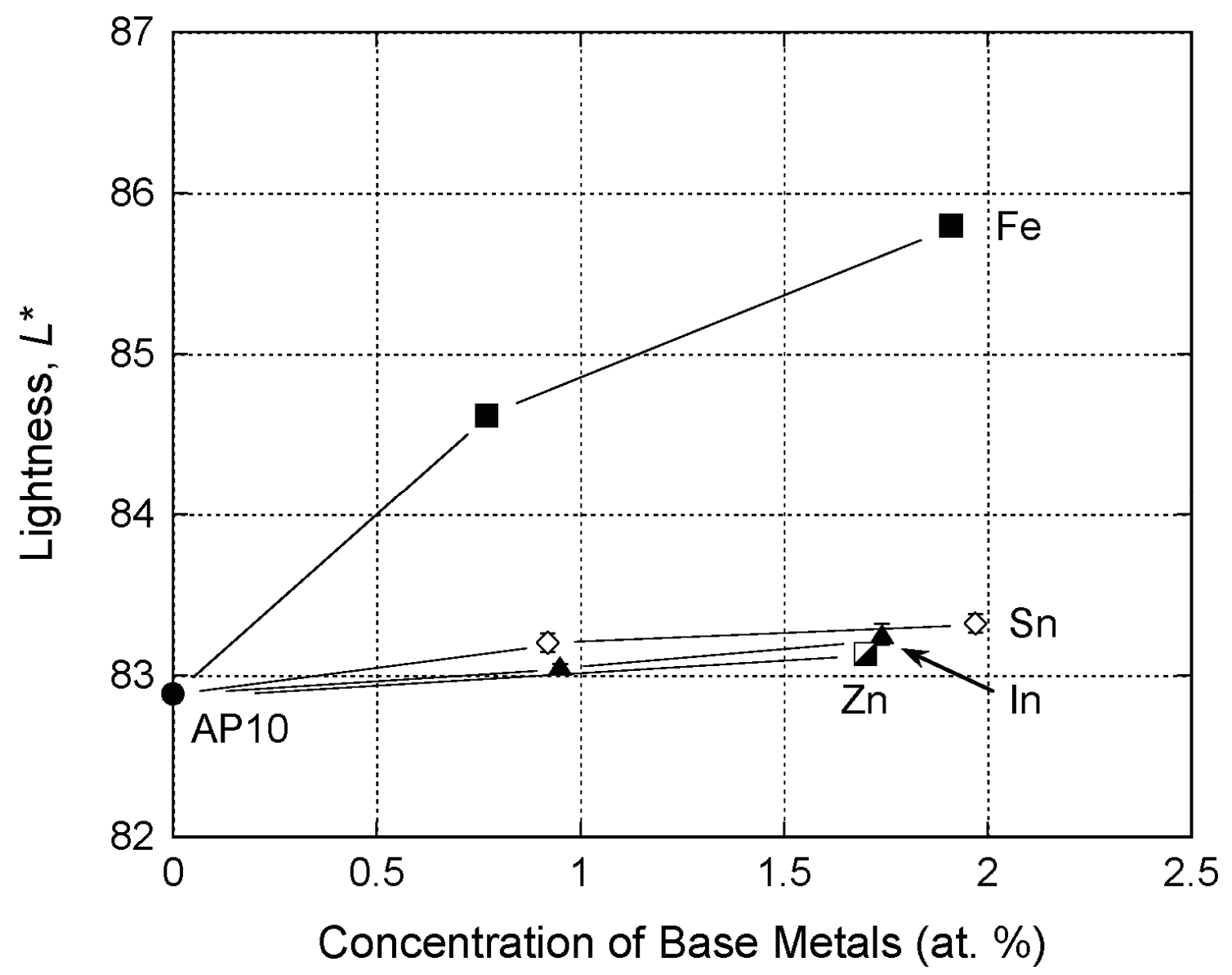

Fig. 8 The effects of alloying addition of the base metals on lightness $L^{*}$ of the binary alloy AP10. 


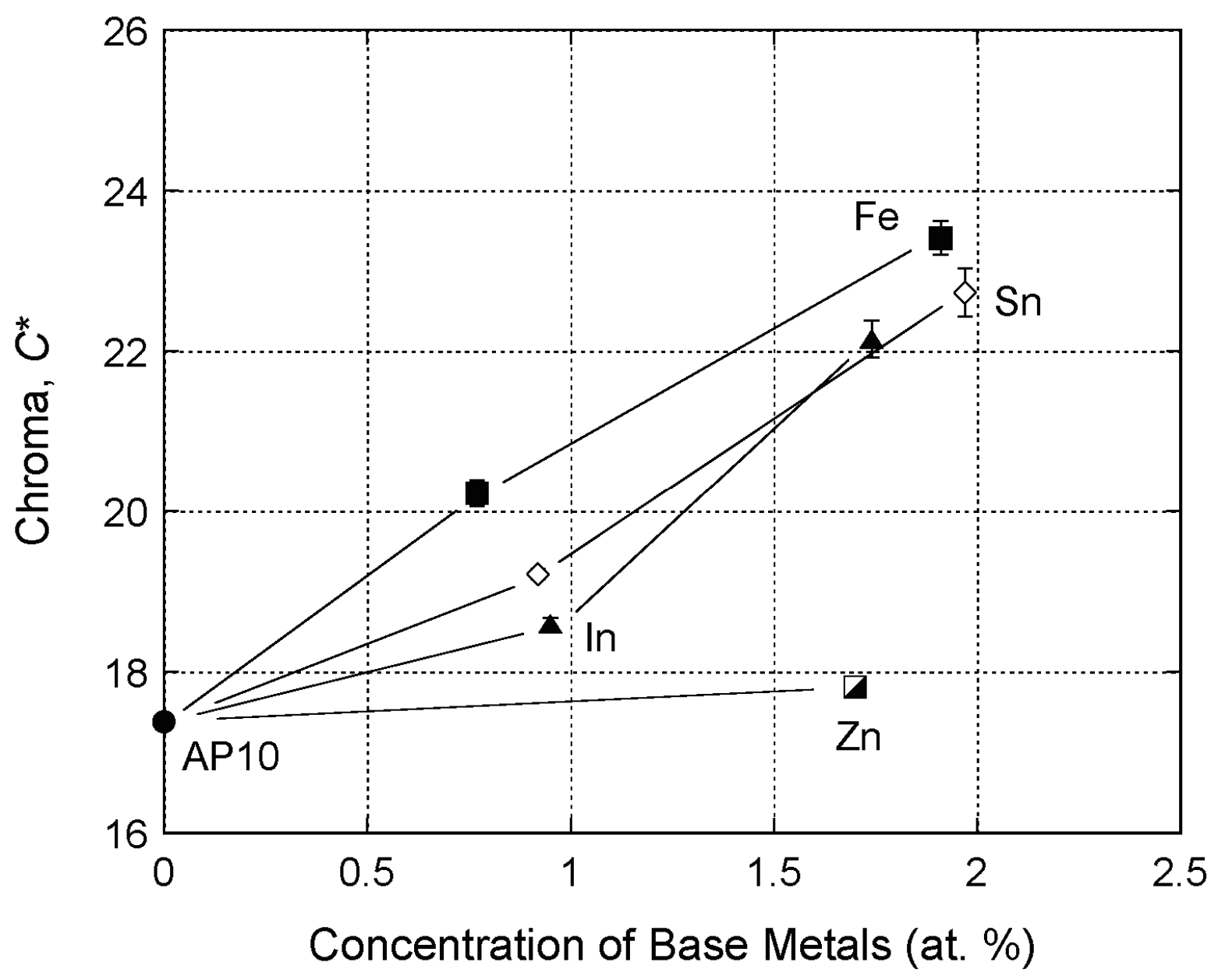

Fig. 9 The effects of alloying addition of the base metals on chroma $C^{*}$ of the binary alloy AP10. 


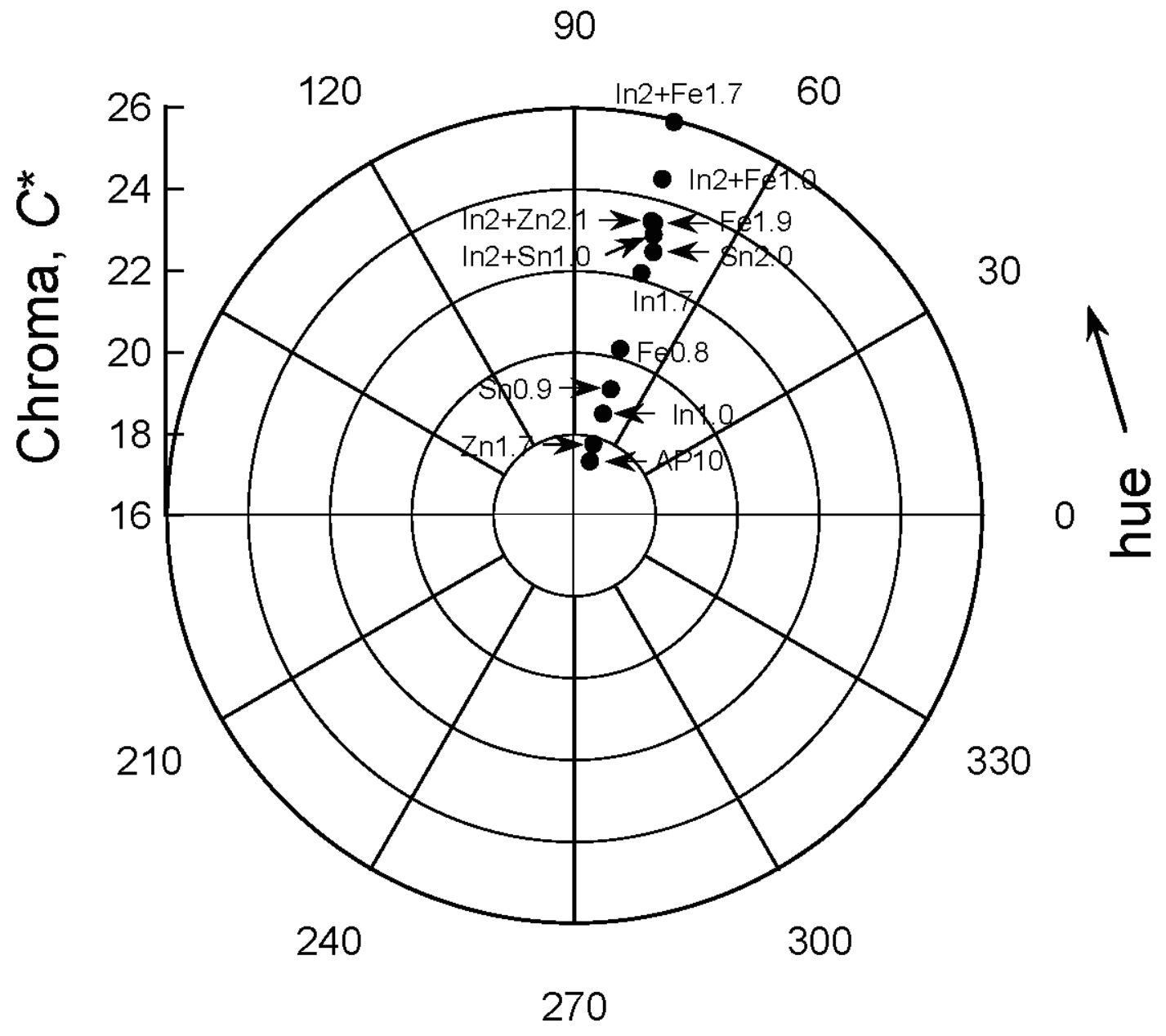

Fig. 10 Distribution of chroma, $C^{*}$, and hue angle, $h$, for all the alloys examined. 


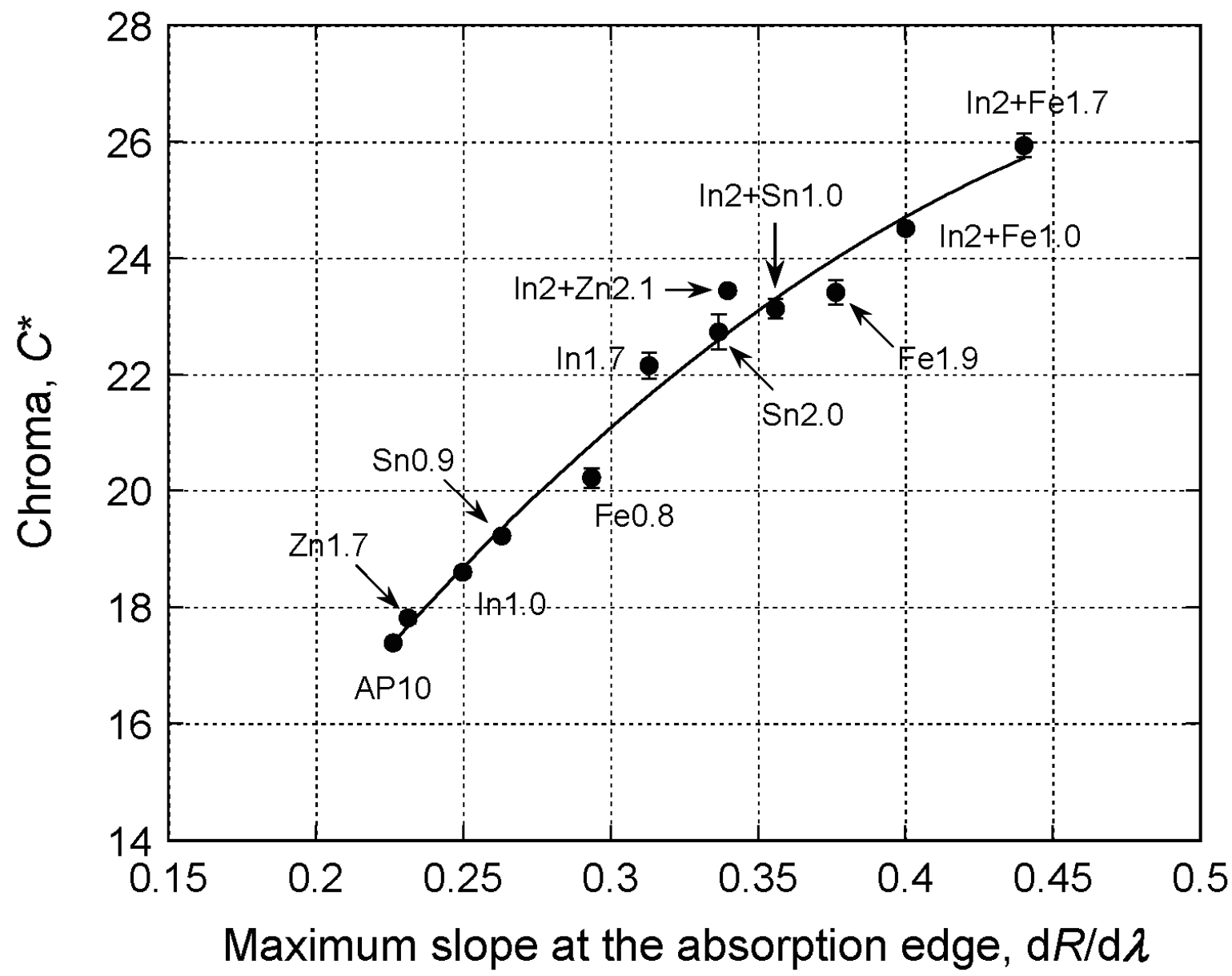

Fig. 11 Relationship between chroma, $C^{*}$, and maximum slope of spectral reflectance curve, $\mathrm{d} R / \mathrm{d} \lambda$, at its absorption edge near $515 \mathrm{~nm}$ for all the experimental alloys. 


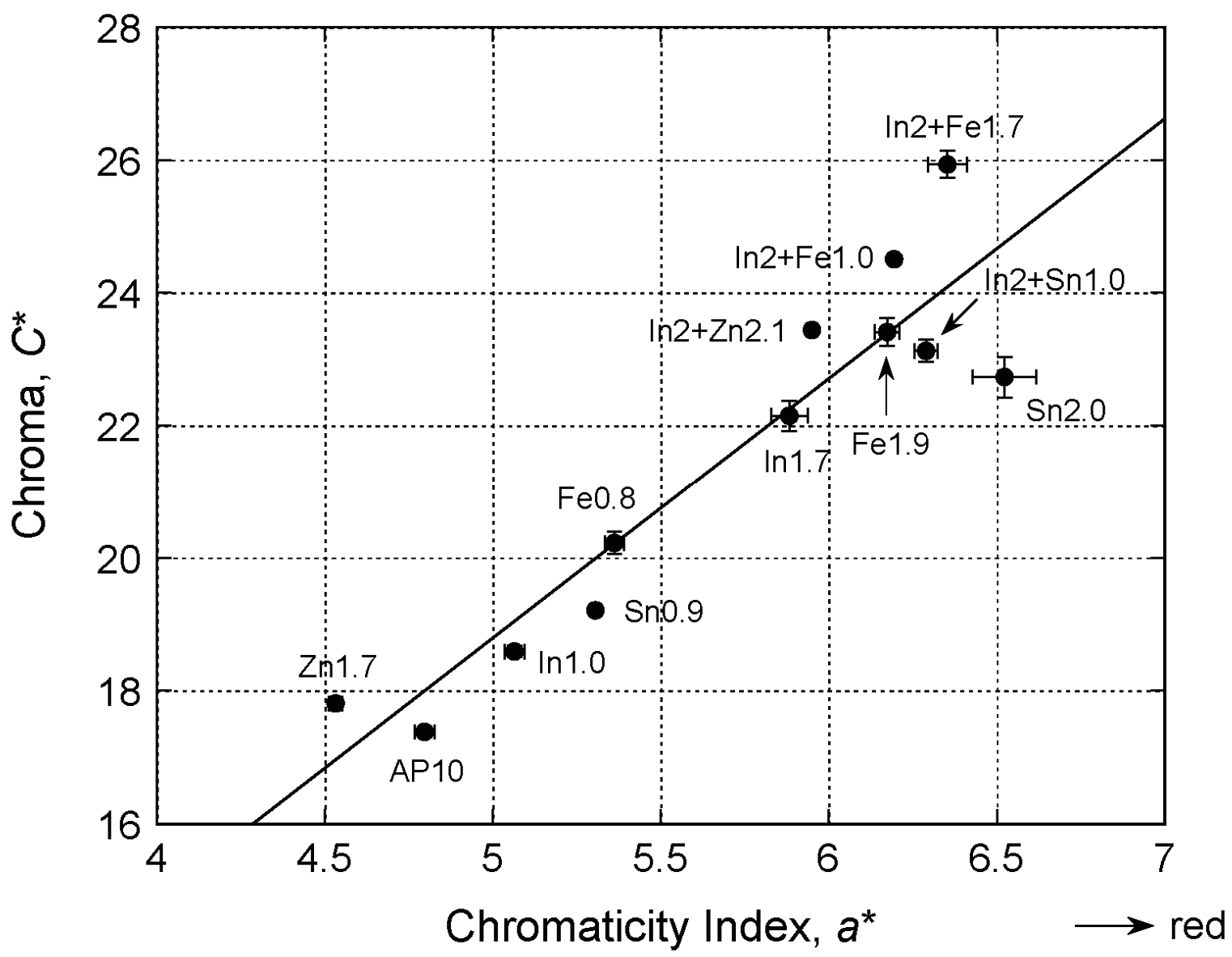

Fig. 12a Relationship between chroma, $C^{*}$, and red-green chromaticity index, $a^{*}$, for all the alloys examined. A correlation coefficient for the regression curve was 0.9290 . 


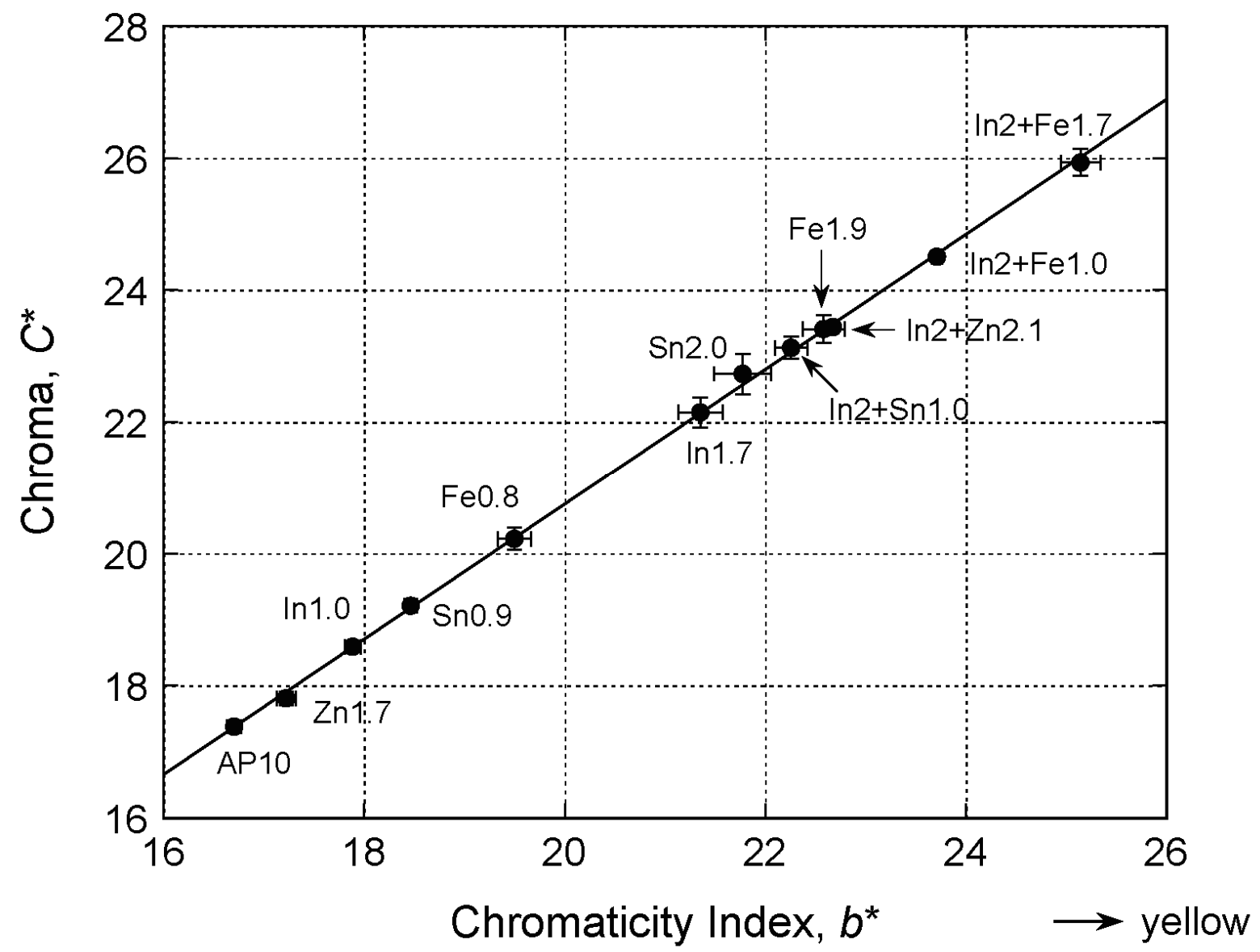

Fig. 12b Relationship between chroma, $C^{*}$, and yellow-blue chromaticity index, $b^{*}$, for all the alloys examined. A correlation coefficient for the regression curve was 0.9997. 


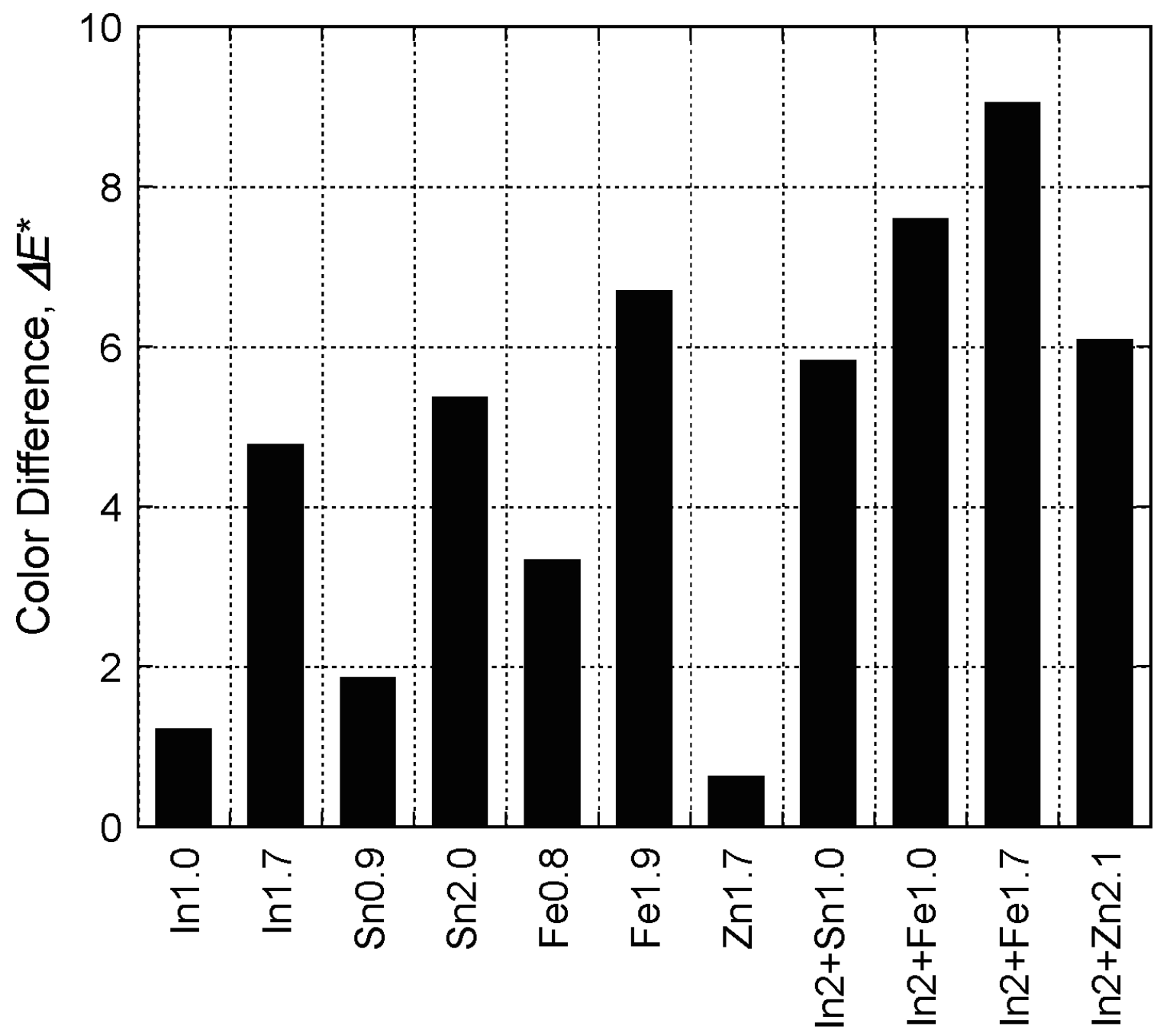

Fig. 13 Color difference, $\Delta E^{*}$, between the reference alloy AP10 and the other experimental alloys. 
Table 1 Chemical compositions of the alloys examined (analyzed values)

\begin{tabular}{|c|c|c|c|c|c|c|c|c|}
\hline \multirow[t]{2}{*}{ System } & \multirow[t]{2}{*}{ Code } & \multicolumn{6}{|c|}{ Composition (at.\%) } & \multirow{2}{*}{$\begin{array}{l}\text { Pt/Au } \\
\text { Ratio }\end{array}$} \\
\hline & & $\mathrm{Au}$ & $\mathrm{Pt}$ & In & $\mathrm{Sn}$ & $\mathrm{Fe}$ & $\mathrm{Zn}$ & \\
\hline Binary alloy & AP10 & 90.11 & 9.89 & 0 & 0 & 0 & 0 & 0.110 \\
\hline \multirow{7}{*}{$\begin{array}{l}\text { Ternary } \\
\text { alloys }\end{array}$} & $\operatorname{In} 1.0$ & 89.12 & 9.93 & 0.95 & 0 & 0 & 0 & 0.111 \\
\hline & In1.7 & 88.35 & 9.91 & 1.74 & 0 & 0 & 0 & 0.112 \\
\hline & $\mathrm{Sn} 0.9$ & 89.15 & 9.93 & 0 & 0.92 & 0 & 0 & 0.111 \\
\hline & $\operatorname{Sn} 2.0$ & 88.22 & 9.81 & 0 & 1.97 & 0 & 0 & 0.111 \\
\hline & $\mathrm{Fe} 0.8$ & 89.19 & 10.04 & 0 & 0 & 0.77 & 0 & 0.113 \\
\hline & Fe1.9 & 88.31 & 9.78 & 0 & 0 & 1.91 & 0 & 0.111 \\
\hline & $\mathrm{Zn} 1.7$ & 88.47 & 9.83 & 0 & 0 & 0 & 1.70 & 0.111 \\
\hline \multirow{4}{*}{$\begin{array}{l}\text { Quaternary } \\
\text { alloys }\end{array}$} & $\operatorname{In} 2+\operatorname{Sn} 1.0$ & 87.33 & 9.77 & 1.95 & 0.95 & 0 & 0 & 0.112 \\
\hline & $\operatorname{In} 2+\mathrm{Fe} 1.0$ & 87.26 & 9.72 & 2.01 & 0 & 1.01 & 0 & 0.111 \\
\hline & $\operatorname{In} 2+\mathrm{Fe} 1.7$ & 86.59 & 9.67 & 2.01 & 0 & 1.73 & 0 & 0.112 \\
\hline & $\operatorname{In} 2+Z n 2.1$ & 86.34 & 9.55 & 1.99 & 0 & 0 & 2.12 & 0.111 \\
\hline
\end{tabular}

